\title{
Erratum to: Genetic diversity of Ixodes pavlovskyi and $I$. persulcatus (Acari: Ixodidae) from the sympatric zone in the south of Western Siberia and Kazakhstan
}

\author{
Natalia N. Livanova ${ }^{1,2} \cdot$ Artem Yu. Tikunov $^{2}$. \\ Alexander M. Kurilshikov ${ }^{2} \cdot$ Stanislav G. Livanov $^{1}$ • \\ Nataliya V. Fomenko ${ }^{2,3} \cdot$ Dmitrii E. Taranenko ${ }^{1}$. \\ Anna E. Kvashnina ${ }^{4}$ Nina V. Tikunova ${ }^{2}$
}

Published online: 23 November 2015

(C) Springer International Publishing Switzerland 2015

\section{Erratum to: Exp Appl Acarol (2015) 67:441-456 DOI 10.1007/s10493-015-9947-7}

Due to an unfortunate turn of events, the figure captions of the above-mentioned publication were transposed. The correct representation of all figures and their captions are published on the following pages and should be treated as definitive (Figs. 1, 2, 3, 4, 5, 6).

The online version of the original article can be found under doi:10.1007/s10493-015-9947-7.

Natalia N. Livanova

nata-livanova@yandex.ru

1 Institute of Systematics and Ecology of Animals Siberian Branch of the Russian Academy of Sciences, Frunze Street 11, 630091 Novosibirsk, Russia

2 Institute of Chemical Biology and Fundamental Medicine of Siberian Branch of the Russian Academy of Sciences, Prosp. Lavrent'eva 8, Novosibirsk, Russia

3 JSC Vector-Best, Pasechna Street 3, Novosibirsk, Russia

4 Federal Nature Preserve "Denezhkin Kamen”, Severouralskiy, Russia 


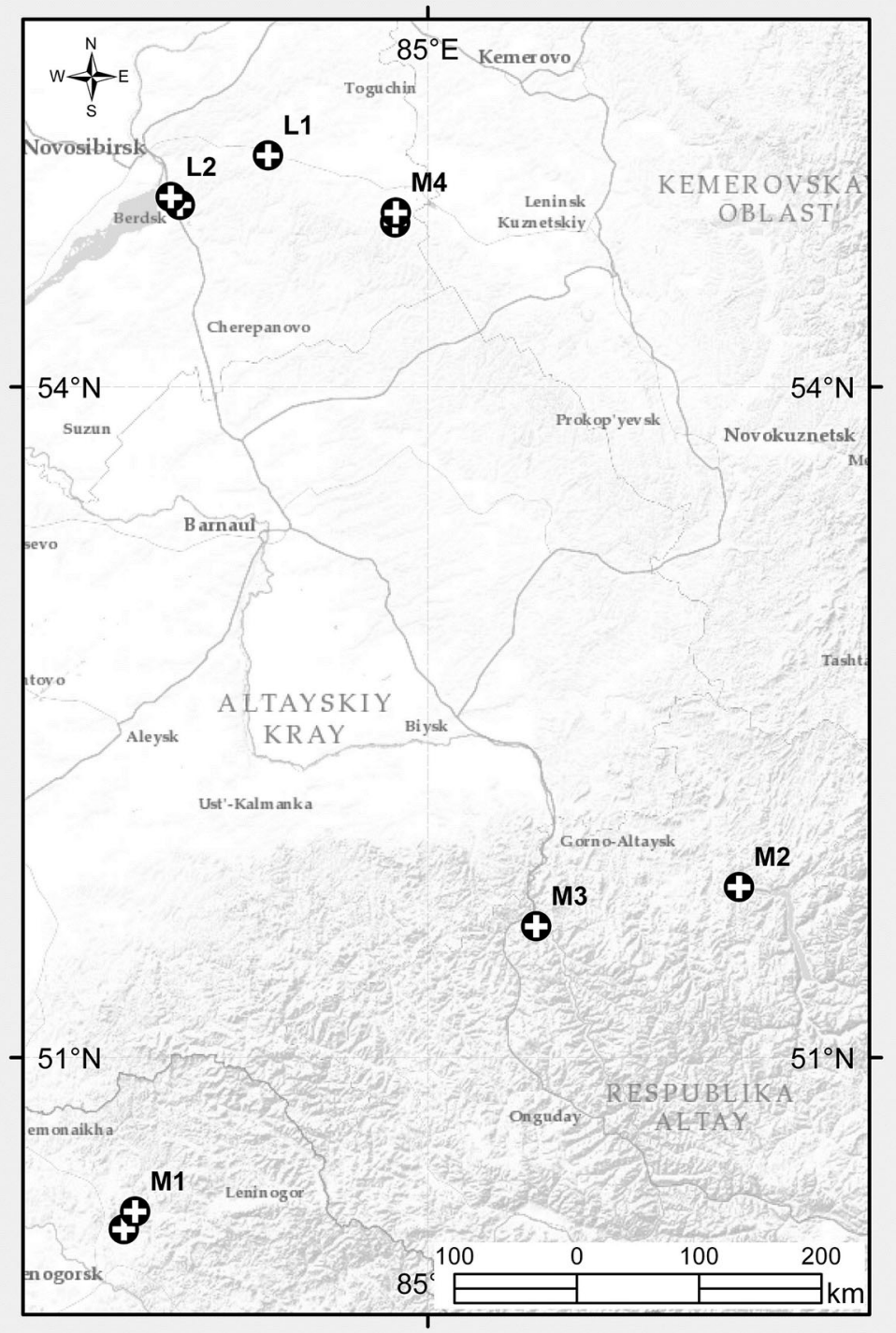

Fig. 1 Sampling sites of Ixodes persulcatus and I. pavlovskyi ticks in different locations of Western Siberia (Russia, Kazakhstan): Western Altai (M1, Kazakhstan), Northeastern Altai (M2, Republic of Altay), and Northern Altai (M3, Republic of Altay), Salair Ridge foothills (M4, Novosibirsk oblast); the forest-steppe of the North of Toguchin (L1) region and the parks near Novosibirsk (L2) 

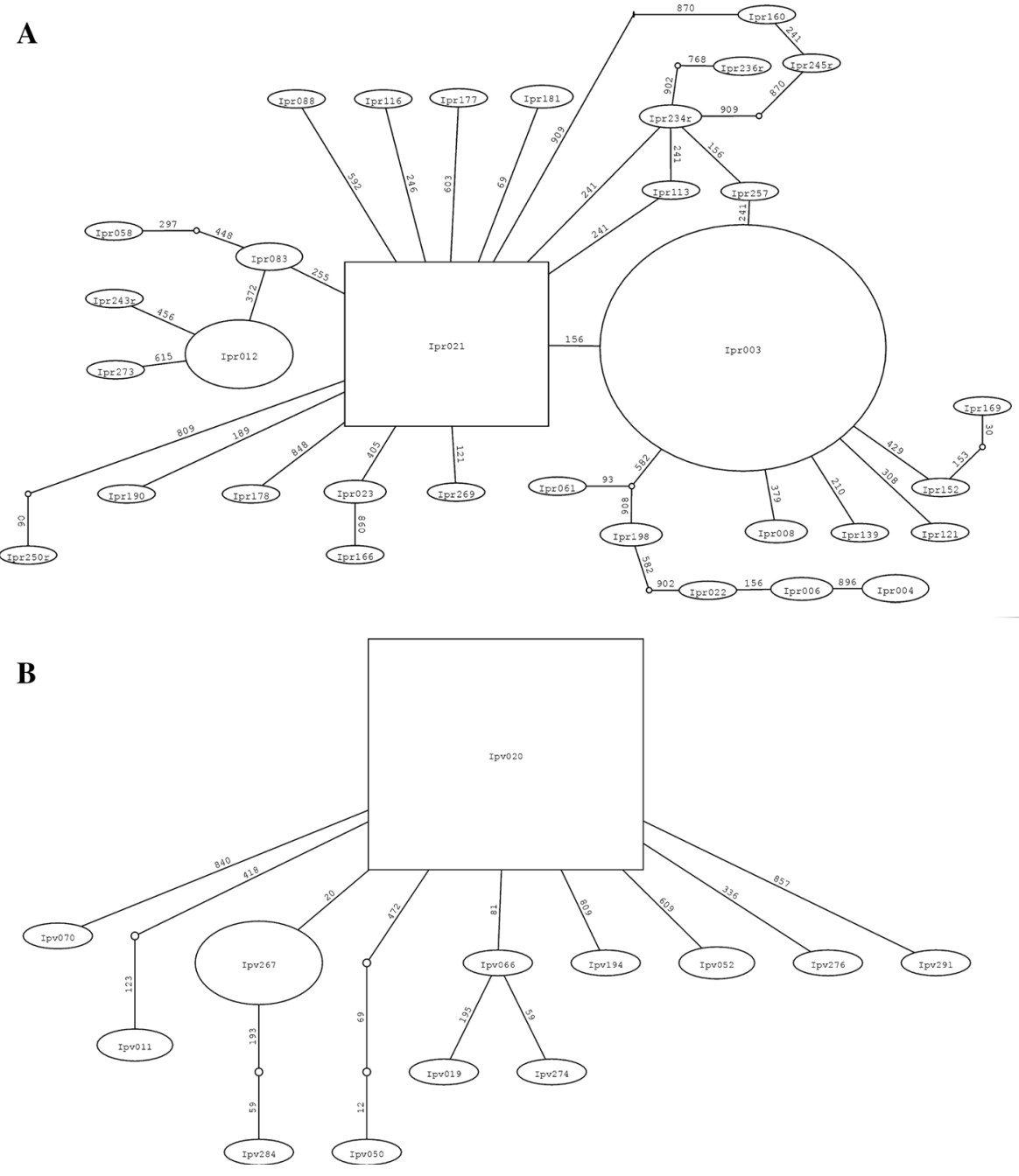

Fig. 2 Haplotype network for Ixodes persulcatus (a) and I. pavlovskyi (b) ticks. Branch labels mean the position in alignment that divides two haplotypes. In total, 33 haplotypes were found in I. persulatus populations studied. Haplotypes were picked based on the concatenated sequences of $16 \mathrm{~S}$ and COI genes 

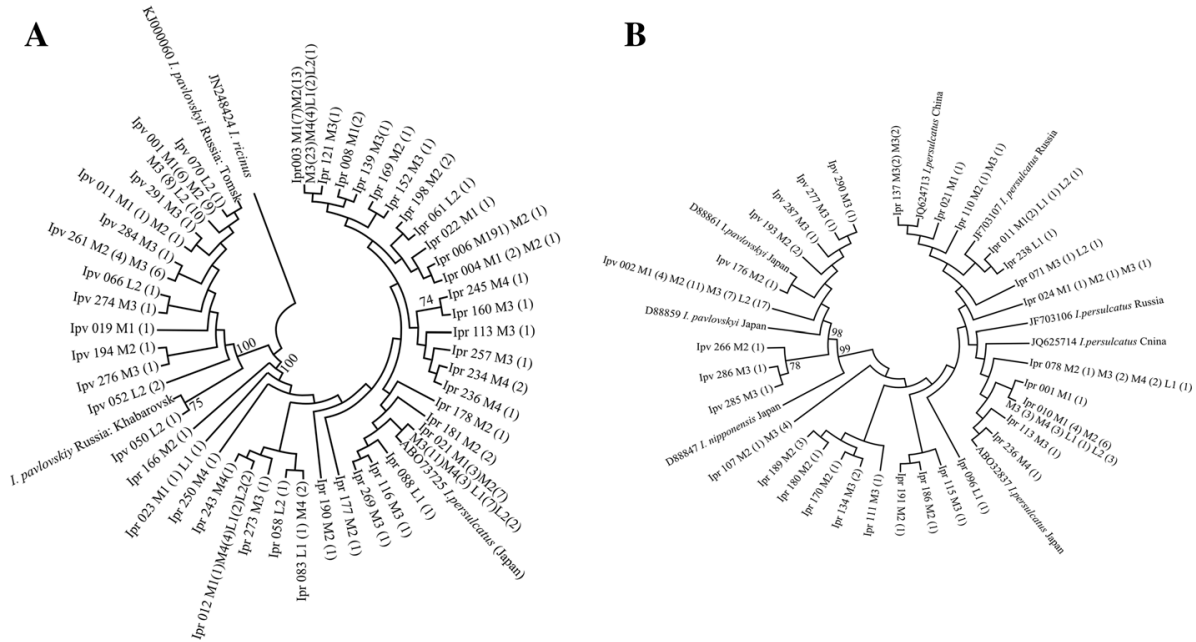

Fig. 3 Neighbor-joining tree for mt 16S rRNA and COI haplotypes (a) and ITS2 (b) of Ixodes persulcatus and I. pavlovskyi. Ixodes ricinus (a) and I. nipponensis (b) were used as outgroups

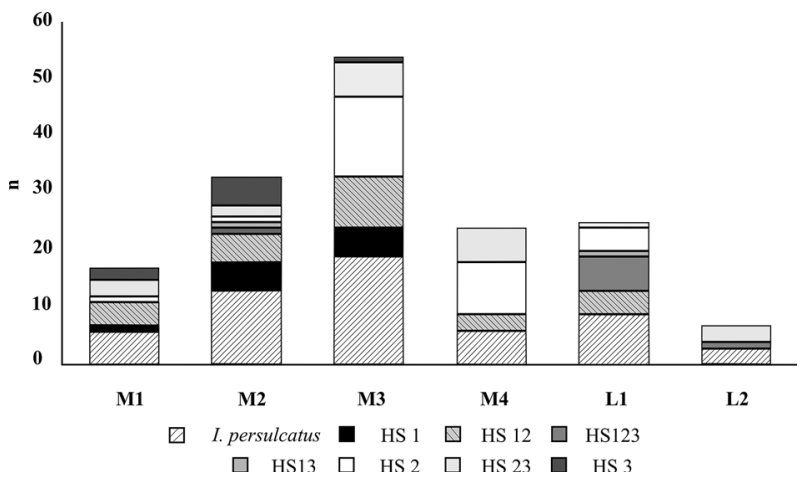

Fig. 4 Distribution of Ixodes persulcatus ticks with different combinations of heterogeneity sites in sequence 


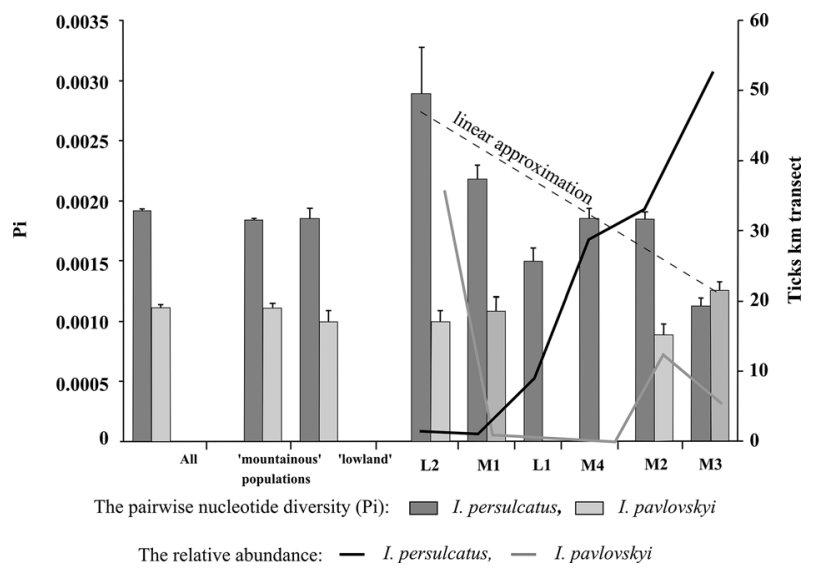

Fig. 5 Pairwise nucleotide diversity $(\mathrm{Pi})$ of the two concatenated mitochondrial gene sequences in relationship with the relative abundance (ticks/km transect) in Ixodes persulcatus, and I. pavlovskyi populations

A
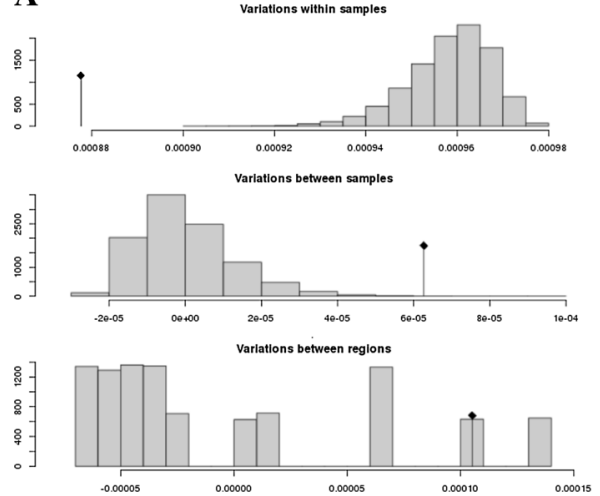
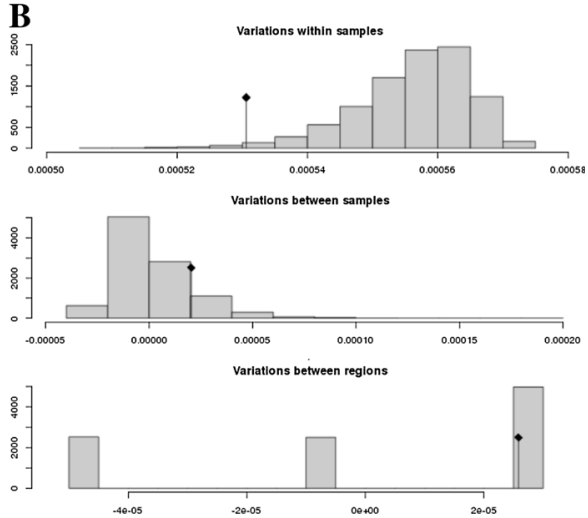

Fig. 6 Histogram of permutated statistics of AMOVA for Ixodes persulcatus (a) and I. pavlovskyi (b) ticks. Vertical line with diamond signifies the value of real sigma 